\title{
Cortical activation to indoor versus outdoor scenes: an fMRI study
}

\author{
John M. Henderson • Christine L. Larson • \\ David C. Zhu
}

Received: 16 August 2006 / Accepted: 16 October 2006 / Published online: 23 November 2006

(C) Springer-Verlag 2006

\begin{abstract}
Prior studies identify two cortical areas, posterior parahippocampal cortex and retrosplenial cortex, that preferentially activate to images of realworld scenes compared to images of other meaningful visual stimuli such as objects and faces. Behavioral and computational studies suggest that sub-categories of real-world scenes differ in their visual and semantic properties. It is presently unknown whether the cortical areas that have been implicated in scene analysis similarly activate differentially to behaviorally relevant scene sub-categories. To examine this issue, we directly compared cortical activation to indoor and outdoor scenes in an fMRI study with a large number of nonrepeated images in each condition. Activation in posterior parahippocampal cortex, including parahippocampal place area, was significantly greater for indoor than outdoor scenes. In contrast, no such difference was observed in retrosplenial cortex, though this region preferentially activated to scenes over faces. These findings suggest differences in function in these two areas. The results are consistent with the view that posterior parahippocampal cortex is functional in processing local space.
\end{abstract}

Keywords Scene perception - Real-world scenes · fMRI $\cdot$ Parahippocampal place area .

Parahippocampal gyrus $\cdot$ Retrosplenial cortex

J. M. Henderson ( $\square)$

Psychology, University of Edinburgh,

7 George Square, Edinburgh EH8 9JZ, UK

e-mail: john.m.henderson@ed.ac.uk

C. L. Larson · D. C. Zhu

Michigan State University, East Lansing, USA

\section{Introduction}

A real-world scene can be defined as a human-scaled view of the $3 \mathrm{D}$ environment within which we are embedded (Henderson and Hollingworth 1999; Henderson and Ferreira 2004). There is growing evidence that real-world scenes differ in important ways from other types of meaningful visual stimuli such as objects, faces, and text (Henderson 2005). For example, scenes contain important regularities in spatial structure and semantics that are not present in other types of visual stimuli (Biederman et al. 1982; Henderson and Hollingworth 1999). From a processing perspective, scenes are recognized more quickly than would be expected on the basis of an analytical route involving recognition of individual objects and their spatial relationships (Intraub 1981; Potter 1976; see also Li et al. 2002; Thorpe et al. 1996), and scenes can be quickly recognized on the basis of scene-level cues even when individual objects cannot be visually resolved (Schyns and Oliva 1994).

An important question in visual cognition is how real-world scene analysis is implemented in the human brain. There are at least three reasons for being concerned with this issue. First, the nature of the neural implementation of scene processing can provide evidence about whether scenes are "special", with identification of scene-specific or scene-preferential cortical areas providing support for the hypothesis that scenes are treated differently than other types of visual stimuli. Second, if scene-specific areas can be identified, then these areas can be queried to determine which features they support. The answers to these queries can then be used to inform functional-level theories of scene perception. Finally, cognitive representations and processes 
ultimately must be implemented by neural systems, and understanding the nature of this implementation is a central concern in cognitive neuroscience.

Results from several fMRI studies suggest the existence of at least two scene processing areas. One area in the posterior region of parahippocampal cortex (pPHC) known as the parahippocampal place area (PPA) has been shown to be selectively activated by scenes over faces, single objects, and object arrays (Bar and Aminoff 2003; Epstein et al. 1999, 2003; Epstein and Kanwisher 1998; see also Aguirre and D'Esposito 1997; Kohler et al. 2002; Maguire et al. 2001). Similarly, there is evidence that retrosplenial cortex preferentially activates to real-world scenes over faces and objects (Bar and Aminoff 2003). Although past studies suggest that pPHC and RSC are involved in real-world scene analysis, it is as yet unclear what specific functions these areas support, and whether the functions diverge for the two regions.

Indoor and outdoor scenes differ in their visual and semantic properties (Oliva and Schyns 2000; Torralba and Oliva 2003; Vailaya et al. 1998). In the present study we asked whether either or both pPHC and RSC similarly reflect this scene-category distinction. The indoor versus outdoor contrast is interesting for several reasons. First, as noted above, the degree to which purported scene regions in the brain reflect computationally and behaviorally important scene distinctions is currently poorly understood. Relatedly, it is unclear whether pPHC and RSC reflect different aspects of scene processing. If they do, then understanding how these areas differ can be used to inform functional-level theories of real-world scene processing. That is, we can investigate the degree to which RSC and pPHC respond similarly or differently to particular scene manipulations as a way to identify functionally important features of scene analysis. Finally, a specific hypothesis about $\mathrm{pPHC}$ function is that it is involved in computing or representing 3D scene geometry. If this hypothesis is correct, then indoor scenes that explicitly depict 3D geometry should lead to greater activation than outdoor scenes that do not. The few fMRI studies that have examined brain activation to indoor and outdoor scenes have produced inconclusive results. In an initial study, Epstein and Kanwisher (1998) reported no statistically significant activation difference in PPA to pictures of rooms versus landscapes. Numerically there was an advantage for rooms over landscapes, but also a numerical advantage for outdoor scenes with landmarks over rooms. Bar and Aminoff (2003) reported a tendency toward an advantage for indoor over outdoor scenes in pPHC, though inferential statistics for this contrast were not provided.
The previous fMRI studies that have directly contrasted indoor and outdoor scenes have tended to use small numbers of repeated scene images. It is known that activation in fMRI studies declines with image repetition (Grill-Spector et al. 1999; Grill-Spector and Malach 2001), an effect that extends to scene processing areas (Epstein et al. 1999, 2003). Therefore, it may be that inconsistencies and null results in past studies were due to image repetition. In the present study, to circumvent any reduction in activity associated with repetition, we adopted a new method in which each subject saw 160 unique scenes in each of the indoor and outdoor conditions in a block-design fMRI experiment. The use of larger image sets also better supports generalization of the results to the population of scenes sampled in each condition. As a control condition, we included an equal number of face photographs. Because we were interested in scene perception and interpretation rather than intentional memory encoding or retrieval, participants passively viewed the images (Goh et al. 2004).

In summary, we report a new fMRI investigation of the cortical processing of indoor versus outdoor scenes in which we directly compared activation to a large number of scene photographs reflecting these scene categories (Fig. 1). Indoor scenes depicted rooms in a house, and outdoor scenes depicted larger-scale outdoor environments. Images were chosen to maximize the features previously shown to distinguish these scene categories (Torralba and Oliva 2003).

\section{Methods}

Subjects

Twenty-one right-handed, healthy Michigan State University students naïve to the stimuli participated in the study. All volunteers signed consent forms approved by the Michigan State University Institutional Review Board. Data from one subject were discarded due to abnormal brain anatomy. Data from three subjects were discarded due to the lack of activation in primary visual areas, suggesting that these subjects were not attending to the display during the study. For the same reason, data from one out of the four functional runs were also discarded in two subjects. A total of 17 subjects ( 7 males and 10 females, mean age 20 , range 18-26) were included in the data analysis.

\section{Stimuli}

Stimuli were 480 full-color digitized photographs selected from a variety of sources, with 160 unique 
pictures for each of the 3 conditions (Fig. 1). A variety of environments were presented for both the indoor and outdoor scenes. The indoor scenes included kitchens, dining rooms, living rooms, and several other types of rooms. Some scenes had a central object as the focus, some were close-up views, and others were wideangle views. This composition is similar to that of the outdoor scenes, which included a variety of types of images, including mountains, seas, lakes, and greenery, among others. As with the indoor photographs, some outdoor scenes were close-up views, others wide-angle. Faces were selected from the AR Face Database (Martinez and Benavente 1998). Stimuli were displayed in color on a $640 \times 480$ LCD monitor mounted on top of the RF head coil. The LCD subtended $12^{\circ} \times 16^{\circ}$ of visual angle.

\section{Procedure}

A block-design paradigm was controlled by an IFISSA system (Invivo Corp., Gainesville, FL). The experiment was divided into four functional runs each lasting $8 \mathrm{~min}$ and $15 \mathrm{~s}$. In each run, subjects were presented with 12 blocks of visual stimulation after an initial $15 \mathrm{~s}$ "resting" period. In each block, ten unique pictures from one condition were presented. Within a block, each picture was presented for $2.5 \mathrm{~s}$ with no inter-stimulus interval. A $15 \mathrm{~s}$ baseline condition (a white screen with
Fig. 1 Examples of the pictures used in the outdoor scene (left column) and indoor scene (right column) conditions. A set of 160 unique scenes were presented without repetition to each subject in each condition, along with an equal number of face control stimuli
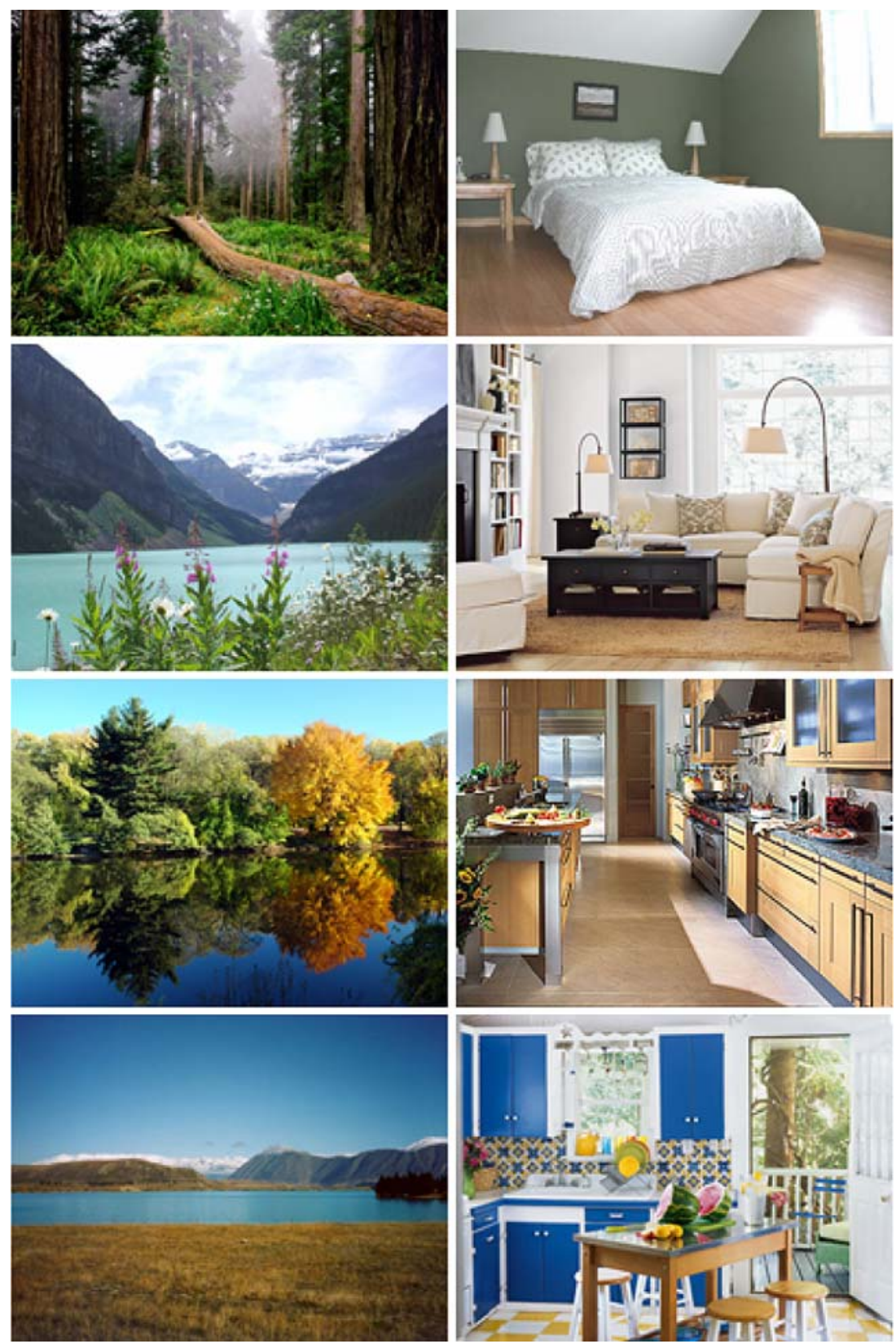
a black cross at the center) followed each block. Each condition was shown in four blocks per run. Both the order of conditions within each run and the order of pictures within a block were initially randomly determined. The four runs were presented to nine subjects in a forward order and eight in a reverse order.

Image acquisition

The experiment was conducted on a 3T GE Signa EXCITE scanner (GE Healthcare, Milwaukee, WI) with an eight-channel head coil. During each session, images were first acquired for the purpose of localization, and first and higher order shimming procedures were then carried out to improve magnetic field homogeneity (Kim et al. 2002). Echo planar images, starting from the most inferior regions of the brain, were then acquired to study brain function with these parameters: 34 contiguous $3 \mathrm{~mm}$ axial slices in an interleaved order, $\mathrm{TE}=25 \mathrm{~ms}, \mathrm{TR}=2,500 \mathrm{~ms}$, flip angle $=80^{\circ}, \mathrm{FOV}=$ $22 \mathrm{~cm}$, matrix size $=64 \times 64$, ramp sampling, and with the first four data points discarded. Each volume of slices was acquired 194 times during each of the four functional runs while subjects viewed the pictures, resulting in a total of 776 volumes of images over the course of the entire experiment. After functional data acquisition, high-resolution volumetric $T_{1}$-weighted spoiled gradient-recalled (SPGR) images with cerebrospinal fluid suppressed were obtained to cover the whole brain with $1241.5 \mathrm{~mm}$ sagittal slices, $8^{\circ}$ flip angle and $24 \mathrm{~cm}$ FOV. These images were used to identify anatomical locations.

fMRI data pre-processing and analysis

All fMRI data pre-processing and analysis were conducted with AFNI software (Cox 1996). For each subject, with the first functional image as the reference, rigid-body motion correction was done in three translational and three rotational directions. The amount of motion in these directions was estimated and then the estimates were used in data analysis. For each subject, spatial blurring with a full width half maximum of $4 \mathrm{~mm}$ was applied to reduce random noise (Parrish et al. 2000), and also to reduce inter-subject anatomical variation and Talairach transformation variation during group analysis. For the group analysis, all images were converted to Talairach coordinate space (Talairach and Tournoux 1988) with an interpolation to $1 \mathrm{~mm}^{3}$ voxels.

For the data analysis of each subject, the reference function throughout all functional runs for each picture category was generated based on the convolution of the stimulus input and a gamma function (Cox 1996), which was modeled as the impulse response when each picture was presented. The functional image data acquired was compared with the reference functions using the $3 \mathrm{dDeconvolve}$ software for multiple linear regression analysis and general linear tests (Ward 2002). Multiple linear regressions were applied on a voxel-wise basis for $t$-statistic tests and to find the magnitude change when each picture condition was presented, compared to the reference functions. The equivalent BOLD percent signal change relative to the baseline state was then calculated. General linear tests were also applied on a voxel-wise basis to find the statistical significance of pair-wise comparisons for all the conditions. For the above analysis, in addition to applying the reference functions for the three picture conditions, MRI signal modeling also included the subject motion estimations in the three translational and the three rotational directions, and the constant, linear and quadratic trends for each of the four functional runs.

\section{Whole brain analysis}

Monte Carlo simulation of the effect of matrix and voxel sizes of the imaging volume, spatial correlation of voxels, voxel intensity thresholding, masking and cluster identification was applied to estimate the overall statistical significance with respect to the whole brain (Ward 2000).

After the percent signal change was estimated with respect to each picture condition for each subject, an analysis of variance (ANOVA) was performed over the seventeen subject data sets for group analysis with a mixed-effect two-factor model. Picture condition (three levels) was the first factor and was modeled to provide a fixed effect. Subject was the second factor and was modeled as a random effect. A voxel-wise $P$ value $<5 \times 10^{-4}$ was used as the statistical threshold to identify the active voxels for each picture condition and the differentially active voxels between conditions. The active voxel selection criteria required that the voxels were nearest neighbor and within a cluster size of $142 \mathrm{~mm}^{3}$. Based on application of these criteria to the whole brain, the voxel-wise $P$ value $<5 \times 10^{-4}$ was corrected to be an equivalent of whole-brain corrected $P$ value $<2.37 \times 10^{-3}$.

Parahippocampal place area (PPA) and retrosplenial cortex (RSC) region of interest (ROI) analyses

In Talairach coordinate space, a PPA ROI was identified in each hemisphere for each subject using a combination of functional activation data and previously 
described anatomical boundaries. Regions showing preferential activation to scenes over faces (thresholding with a voxel-wise $P<10^{-4}$ based on the $t$ test of the "Indoor + Outdoor $-2 \times$ Face" condition contrast) in the parahippocampal gyrus were defined as the PPA. This contrast resulted in clear PPA ROIs in posterior parahippocampal cortex in each hemisphere. We computed the average BOLD percent signal change for all voxels in the PPA ROI individually for each subject as a function of condition and hemisphere. These data were entered into a condition (indoor, outdoor, faces) by hemisphere (left, right) ANOVA. We conducted a similar subject analysis for retrosplenial cortex. An analogous condition $\times$ hemisphere ANOVA was calculated for the mean percent signal change by subject for all voxels in the anatomically defined (BA 29 and 30) RSC ROI.

\section{Results}

\section{Whole brain analysis}

An initial whole-brain group analysis contrasted the two scene conditions with the face condition. The results confirmed that regions previously found to show preferential activation to faces (notably fusiform gyrus and amygdala) were more active to faces over scenes, and that the main areas of interest, posterior parahippocampal cortex and retrosplenial cortex, were preferentially active to scenes over faces (Figs. 2a, 3a, b).
These results establish that the experiment was sensitive to image-specific activation, enabling our direct contrast of the indoor and outdoor scene conditions.

In the main group-wise whole-brain contrasts of interest, activation to indoor and outdoor scenes was compared. Figure 2 and Table 1 show the results. Of special interest were the contrasts of the indoor versus outdoor scene conditions in the parahippocampal gyrus and retrosplenial cortex, two regions that have specifically been identified as scene processing areas in past fMRI studies (e.g., Bar and Aminoff 2003; Epstein et al. 1999, 2003; Epstein and Kanwisher 1998).

Considering first the parahippocampal gyrus, activation was greater for the indoor than the outdoor scenes (Fig. 2b, c). This differential activation included the region of posterior parahippocampal cortex typically identified as PPA, with one of the two activation peaks in the right parahippocampal cluster $(26,-47,-4)$ and the activation peak in the left parahippocampal cluster $(-27,-42,-5)$ falling within the cortical region associated with PPA (Epstein and Kanwisher 1998). This activation was observed without restricting the analysis to the PPA a priori. The indoor versus outdoor activation difference was more extensive in the right than the left hemisphere, 2,180 versus 1,204 $\mathrm{mm}^{3}$, respectively. The posterior parahippocampal cortex clusters also contained a small amount of significant activation in adjacent regions, including right fusiform gyrus in the right parahippocampal cluster (Table 1).

In contrast to posterior parahippocampal cortex, no significant difference in activation was observed for the
Fig. 2 The spatial extents of differential activation in the whole-brain group analysis (voxel-wise $t$ value scale on the left) shown through color maps. a The scene versus face contrast in axial and coronal views. b The indoor versus outdoor contrast in axial and coronal views. c The indoor versus outdoor contrast in 3D views. The crosshair in all images indicates the peak $t$ value for the indoor versus outdoor contrast in the right parahippocampal gyrus $(26,-47,-4)$

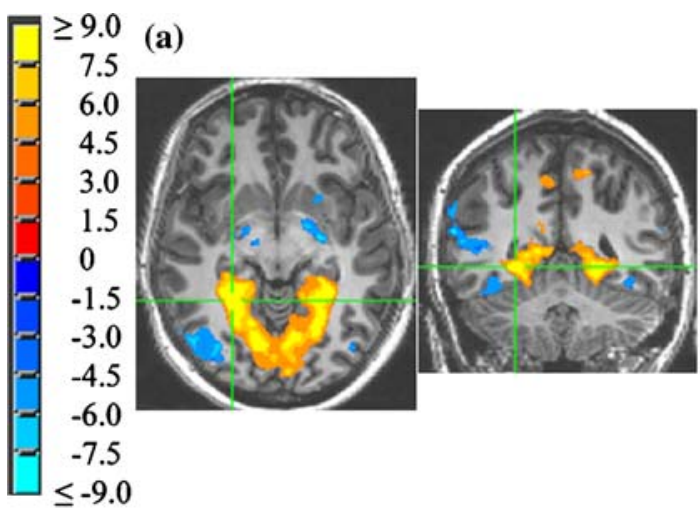

(b)

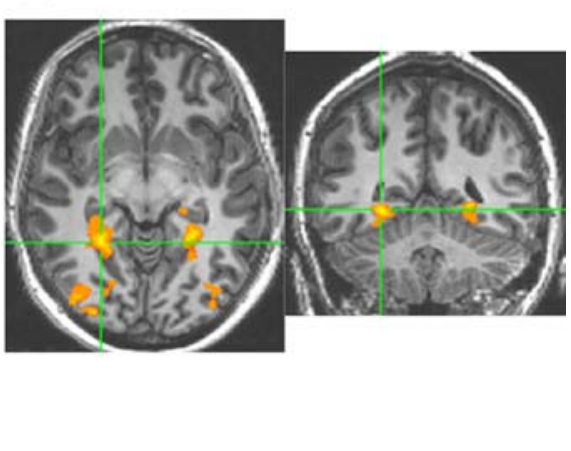

(c)

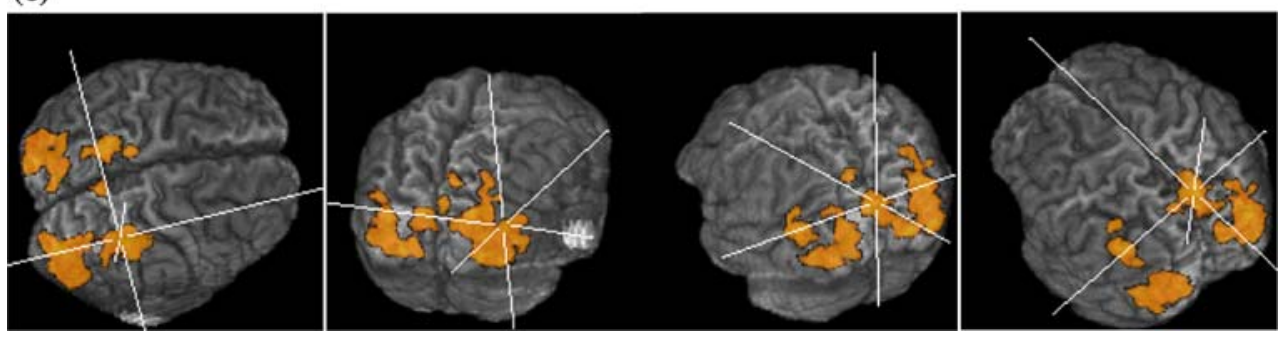


Fig. 3 The differential activation at the retrosplenial cortex in the whole-brain group analysis (voxel-wise $t$ value scale on the left) shown through color maps. a The indoor scene versus face contrast in axial and coronal views. b The outdoor scene versus face contrast in axial and coronal views. c The indoor versus outdoor scene contrast in axial and coronal views. The green crosshair indicates the peak $t$ value for the scene versus face contrast in the right retrosplenial cortex $(17,-58,14)$ (a)

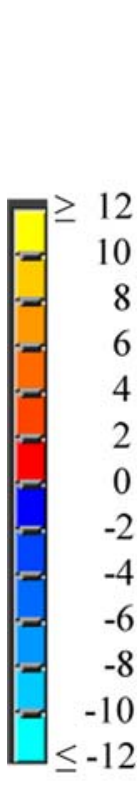

(b)
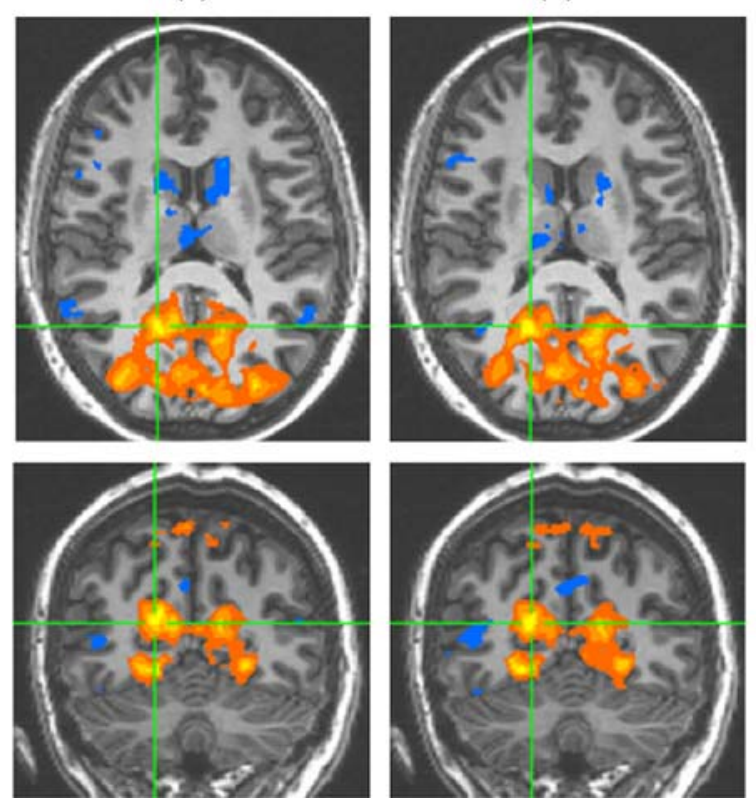

(c)

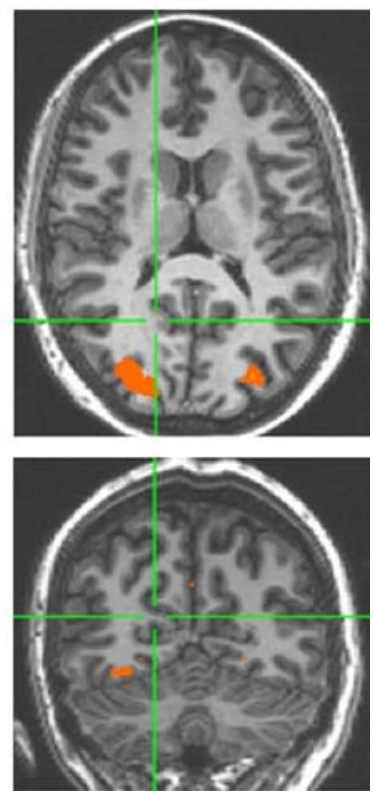

Table 1 Group analysis results for the contrast of indoor versus outdoor scenes by hemisphere

\begin{tabular}{|c|c|c|c|c|c|}
\hline Clusters & $\begin{array}{l}\text { Active } \\
\text { volume } \\
\left(\mathrm{mm}^{3}\right)\end{array}$ & $\begin{array}{l}\text { Max } t \\
\text { values }\end{array}$ & $\begin{array}{l}\text { Max } t \text { value } \\
\text { locations }\end{array}$ & $\begin{array}{l}\text { Signal change } \\
\text { indoor }(\%)\end{array}$ & $\begin{array}{l}\text { Signal change } \\
\text { outdoor }(\%)\end{array}$ \\
\hline Right parahippocampal cluster ${ }^{a}$ & 2,180 & $\begin{array}{l}8.19 \\
8.15\end{array}$ & $\begin{array}{l}(29,-43,-13) \\
(26,-47,-4)\end{array}$ & $1.06 \pm 0.38$ & $0.77 \pm 0.33$ \\
\hline Left parahippocampal cluster ${ }^{b}$ & 1,204 & 7.77 & $(-27,-42,-5)$ & $0.88 \pm 0.25$ & $0.61 \pm 0.21$ \\
\hline Right middle occipital cluster ${ }^{c}$ & 7,031 & 8.74 & $(25,-76,-8)$ & $1.36 \pm 0.56$ & $1.09 \pm 0.54$ \\
\hline Left middle occipital cluster ${ }^{\mathrm{d}}$ & 1,630 & 7.04 & $(-26,-85,8)$ & $1.35 \pm 0.32$ & $1.12 \pm 0.30$ \\
\hline Left lingual/fusiform cluster ${ }^{\mathrm{e}}$ & 2,242 & 7.93 & $(-35,-65,-12)$ & $1.92 \pm 0.74$ & $1.63 \pm 0.73$ \\
\hline
\end{tabular}

Five well-defined clusters were found to be significantly active for this contrast. The reported data for each cluster are: total active volume, maximum $t$ values and their locations (Talairach coordinates), and percent signal change (mean $\pm \mathrm{SD}$ ) for each condition. For each cluster, the anatomical loci of the significantly active region along with their active volumes are also reported

a Includes $1,051 \mathrm{~mm}^{3}$ of right parahippocampal gyrus and $490 \mathrm{~mm}^{3}$ of right fusiform gyrus

b Includes $876 \mathrm{~mm}^{3}$ of left parahippocampal gyrus

c Includes 3,691 $\mathrm{mm}^{3}$ of right middle occipital gyrus, $732 \mathrm{~mm}^{3}$ of right inferior occipital gyrus, $596 \mathrm{~mm}^{3}$ of right lingual gyrus, and $207 \mathrm{~mm}^{3}$ of right precuneus

${ }^{\mathrm{d}}$ Includes $1,079 \mathrm{~mm}^{3}$ of left middle occipital gyrus

e Includes $467 \mathrm{~mm}^{3}$ of left lingual gyrus, $419 \mathrm{~mm}^{3}$ of left fusiform gyrus, $293 \mathrm{~mm}^{3}$ of left middle occipital gyrus, and $268 \mathrm{~mm}{ }^{3}$ of left inferior occipital gyrus

indoor versus outdoor scene contrast in retrosplenial cortex, with non-significant 5 and $6 \mathrm{~mm}^{3}$ active volumes seen in the right and left retrosplenial cortex, respectively (Fig. 3c). Importantly, retrosplenial cortex did show robust preferential activation to scenes when contrasted directly with faces (Fig. 3a, b). Specifically, 1,713 and $1,503 \mathrm{~mm}^{3}$ active volumes were seen in the right and left retrosplenial cortex, respectively, with the indoor scenes versus faces contrast, and 1,599 and $1,350 \mathrm{~mm}^{3}$ active volumes were seen in the right and left retrosplenial cortex, respectively, with the outdoor scenes versus faces contrast.
Finally, although not of specific interest, differential activation was also observed between indoor versus outdoor scenes in three additional clusters. Two of these comprised right and left middle occipital cortex and adjacent structures, and the third comprised a region of left lingual gyrus and fusiform gyrus and adjacent structures (Fig. 2; Table 1).

Region of interest analyses

All 17 subjects showed well-defined PPA ROIs, with an average right PPA ROI volume of $3,651 \mathrm{~mm}^{3}$ (range 
2,426-5,186 $\mathrm{mm}^{3}$ ), and average left PPA ROI volume of 3,390 $\mathrm{mm}^{3}$ (range 2,284-5,263 $\mathrm{mm}^{3}$ ). Peak $t$ values in Talairach coordinates for the right and left hemisphere based on the group analysis were $(25,-50,-4)$ and $(-28,-37,-7)$, respectively. The condition (indoor, outdoor, faces) by hemisphere (left, right) ANOVA for PPA revealed a significant effect of condition, $F$ $(2,32)=360.11, P<0.001, \eta^{2}=0.957$, and a marginally significant effect of Hemisphere, $F(1,16)=4.00$, $P=0.06, \eta^{2}=0.200$ (Fig. 4a). Pair-wise comparisons for the main effect of condition indicated that PPA activation was greatest for indoor scenes, followed by outdoor scenes, with no activation for faces, all $P<0.001$, Bonferroni corrected). The trend for greater right than left PPA activation was qualified by a condition $\times$ hemisphere interaction, $F(2,32)=6.78$, $P=0.01, \eta^{2}=0.298$, which indicated greater right than left PPA activation for indoor and outdoor scenes $(P<0.05$, Bonferroni corrected $)$, but not for faces.

The ROI analysis for RSC (Fig. 4b) revealed a significant main effect of condition, $F(2,32)=84.94$, $P<0.001, \eta^{2}=0.841$. Post-hoc pair-wise comparisons showed that both types of scenes more strongly activated RSC than faces $(P<0.001$, Bonferroni corrected), but that there was no difference between indoor and outdoor scenes $(P>0.65)$. The main effect
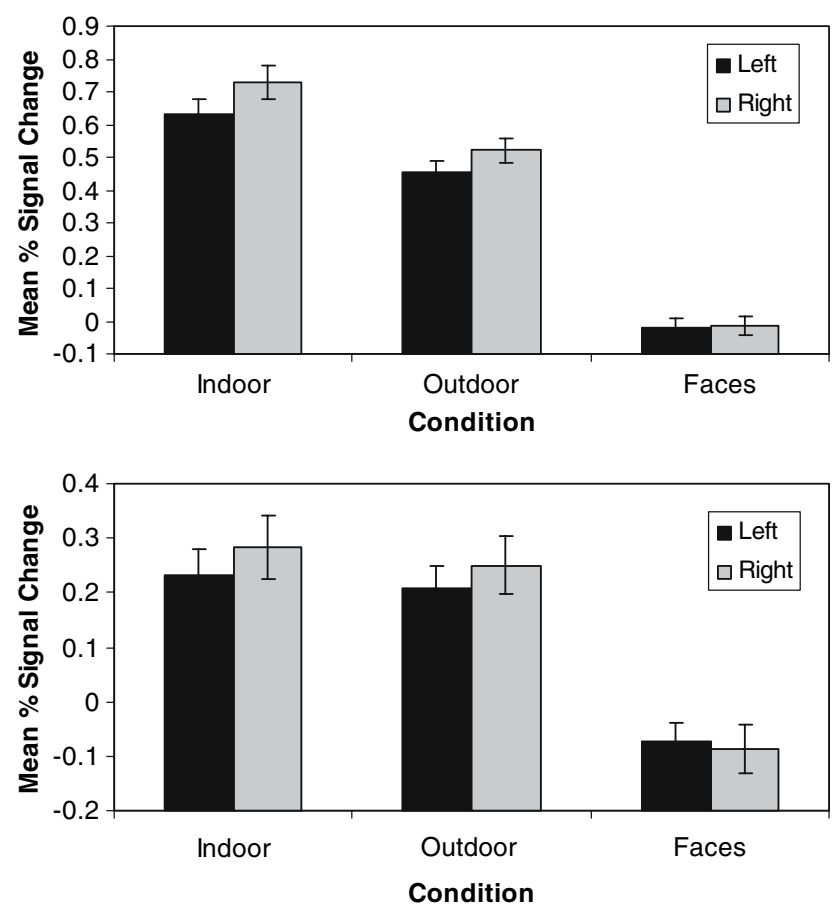

Fig. 4 a Mean percent signal change (and SEM) across all voxels in the PPA ROI for 17 subjects as a function of image condition and hemisphere. b Mean percent signal change (and SEM) across all voxels in the RSC ROI for 17 subjects as a function of image condition and hemisphere for hemisphere was not significant, $F(1,16)=1.52$, $P=0.22$. The scene effect was qualified by a marginal condition $\times$ hemisphere interaction, $F(2,32)=3.62$, $P=0.06, \eta^{2}=0.185$. While there was no hemispheric asymmetry for faces, there was a trend for greater right than left RSC activation for both types of scenes, though neither contrast reached significance ( $p$ s $>0.09$ ).

Confirming the dissociation between patterns of activation in the PPA and RSC, a subsequent condition $x$ hemisphere $\times$ ROI ANOVA revealed a condition $\times$ ROI interaction, $F(2,32)=56.32, P<0.001, \eta^{2}=0.779$. As expected from the previous ANOVAs, this interaction was due to the fact that PPA was more strongly activated by indoor than outdoor scenes $(P<0.001)$, whereas no difference between these conditions was present for the RSC $(P>0.20)$.

\section{Discussion}

The purpose of this study was to investigate the nature of real-world scene processing in the brain. Specifically, we were interested in determining whether two classes of scenes that are known to differ on behavioral and computational grounds are treated differently by previously identified scene processing areas of cortex. Prior studies comparing indoor and outdoor scenes have been inconsistent and inconclusive (Bar and Aminoff 2003; Epstein and Kanwisher 1998), potentially because of lack of power due to stimulus repetition. Using a large set of non-repeated scene images, the present study clearly demonstrates that activation is greater for indoor than outdoor scenes in posterior parahippocampal cortex including PPA, but not in retrosplenial cortex.

One possible explanation for the difference in indoor versus outdoor scene activation in $\mathrm{pPHC}$ is that this region is involved in processing the geometric structure of local 3D space (Epstein 2005; Epstein et al. 2003). This hypothesis predicts that posterior parahippocampal cortex activation should increase as more $3 \mathrm{D}$ geometry becomes explicit in a scene image. Epstein et al. (2003) reported the results of a pilot experiment showing similar activation to tabletop scenes versus landscapes and rooms, a result that is inconsistent with the local geometry hypothesis. However, in a recent study contrasting close-up and full-view scenes, we found that PPA was preferentially activated by fullviews compared to close-up views (Henderson et al. 2006). Because the full-view scenes in that study explicitly depicted local 3D space whereas the close-up scenes did not, these results can be taken to support the local geometry hypothesis. But full-view scenes 
also depict more open space than do close-up scenes; leaving open the alternative hypothesis that posterior parahippocampal cortex prefers more global spatial views. The present results are consistent with the local geometry hypothesis but not the global views hypothesis of posterior parahippocampal function because widening the view from indoor to outdoor did not lead to greater posterior parahippocampal cortex activation. Across studies, then, close-up scenes produced less activation than full-view indoor scenes, but full-view indoor scenes produced more activation than outdoor scenes. Together, these results suggest that local 3D space is the most robust elicitor of posterior parahippocampal cortex activation. These results in turn suggest that one important way in which scenes are special compared to other types of visual stimuli is that they often include local 3D environmental structure.

Indoor and outdoor scenes also differ along a number of dimensions including their image statistics (Oliva and Schyns 2000; Torralba and Oliva 2003; Vailaya et al. 1998), and it is possible that these differences directly drive posterior parahippocampal cortex activation. Some evidence supporting this possibility comes from the preferential activation to indoor versus outdoor scenes observed in the present study in the middle occipital gyrus clusters. Direct manipulation of image statistics associated with indoor versus outdoor scenes will be necessary to determine if differences in image statistics are the direct basis for the observed difference in posterior parahippocampal cortex. The results of the present study indicate that such direct manipulation would be a worthwhile enterprise.

There are several additional potential explanations for the indoor-outdoor activation difference in parahippocampal cortex. One is that indoor scenes are more interesting (and so attentionally engaging) than outdoor scenes. Although a logical possibility, we know of no independent evidence or theoretical motivation that would support this idea. In fact, there is evidence that features of outdoor scenes such as naturalness and openness are preferred over features of indoor scenes such as their artifactual nature (Biederman and Vessel 2006; Vessel and Biederman 2002), potentially because of their value in evolutionary terms (Kaplan 1992). A second potential difference between indoor and outdoor scenes is that indoor scenes (either in general or in our sample) might contain more discrete objects, and the number of individuated objects (or the number of spatial relationships among objects) might be the source of the differential activation in posterior parahippocampal cortex. Contrary to this hypothesis, however, in a direct comparison of PPA response to empty rooms versus furnished rooms versus arrays of objects, Epstein and Kawisher (1998) found that activation was equivalent to empty and furnished rooms, whereas activation to both types of rooms was over twice as great as to the object arrays. At the same time, activation to object arrays was equivalent to single objects. From these results it would appear that the number of objects or spatial relations among objects is not the critical determinant of activation in posterior parahippocampal cortex. Third, the indoor versus outdoor distinction as operationalized here is also potentially captured by the distinction between man-made versus natural scenes. There is some computational evidence that image statistics provide a basis for categorizing scenes on the naturalness dimension (Vailaya et al. 1998). Whether the observed indoor-outdoor differences observed here are better thought of as arising from spatial structural differences more comfortably related to an indoor-outdoor contrast, or instead are better thought of as arising from features associated with a man-made versus natural contrast, is a question that we are currently actively pursuing. An answer to this question will further constrain functional-level theories of scene perception, specifying the dimensions along with scenes differ functionally from other types of visual stimuli.

In comparison to posterior parahippocampal cortex, retrosplenial cortex showed no significant preferential activation to either indoor or outdoor scenes, though this region did show robust preferential activation to scenes compared to faces. Prior studies have suggested that retrosplenial cortex plays a role in spatial navigation or orientation to large-scale space (Maguire 2001), for example converting between egocentric and allocentric spatial representations (Burgess et al. 2001) or integrating egocentric spatial information with selfmotion cues (Wolbers and Buchel 2005). From this perspective, one might expect greater retrosplenial activation to outdoor than indoor scenes, a result that was clearly not observed here. However, it is possible that the passive viewing task used in the present study does not engage navigational processing. A second hypothesis concerning retrosplenial cortex proposes that it is involved in the analysis of scene-relevant associations between objects or between objects and scene categories (Bar 2004; Bar and Aminoff 2003). On this hypothesis, differential activation to indoor and outdoor scenes in retrosplenial cortex would not necessarily be expected, consistent with the current results.

\section{Conclusions}

In summary, the present study demonstrates that a bilateral posterior region of parahippocampal cortex 
known to be involved in high-level scene processing is preferentially activated by indoor over outdoor scenes. These results converge with computational and behavioral evidence indicating that the indoor-outdoor distinction constitutes a functional scene-class boundary. The results provide the first direct evidence that a behaviorally relevant scene class distinction is reflected in at least one scene processing region in the ventral visual stream. The results also demonstrate dissociation in function between two scene processing areas, with pPHC responding to the indoor-outdoor distinction and RSC showing equivalent activation to these two stimulus classes. Indoor and outdoor scenes differ along a number of dimensions including image statistics, spatial structure, and semantic interpretation. An important goal for future research will be to determine whether image statistics related to spatial structure can drive the observed activation difference in $\mathrm{PPHC}$ in the absence of direct spatial or semantic cues.

Acknowledgments This research was supported by grants from the MSU Foundation to John M. Henderson, and from the MSU Intramural Research Grant Program and NIMH (MH071275-01) to Christine L. Larson. We also thank Michigan State University's Department of Radiology, Department of Psychology, Office of the Vice President for Research and Graduate Studies, and Core Analytical Fund for their support. We thank David McFarlane, Jie Huang, Jeremy Grounds, Shawn Katterman, Stephen Kemsley, and Jordan Robinson for their assistance on the project, and Moshe Bar for his constructive comments on an earlier draft of this article.

\section{References}

Aguirre G, D'Esposito M (1997) Environmental knowledge is subserved by separate dorsal/ventral areas. J Neurosci 17:2512-2518

Bar M (2004) Visual objects in context. Nat Rev Neurosci 5:617629

Bar M, Aminoff E (2003) Cortical analysis of visual context. Neuron 38:347-358

Biederman I, Vessel E (2006) Perceptual pleasure and the brain. Am Sci 94:248-255

Biederman I, Mezzanotte RJ, Rabinowitz JC (1982) Scene perception: detecting and judging objects undergoing relational violations. Cognit Psychol 14:143-177

Burgess N, Becker S, King JA, O'Keefe J (2001) Memory for events and their spatial context: models and experiments. Philos Trans R Soc Lond B 356:1493-1503

Cox RW (1996) AFNI: software for analysis and visualization of functional magnetic resonance images. Comput Biomed Res 29:162-173

Epstein R (2005) The cortical basis of visual scene processing. Vis Cogn 12:954-978

Epstein R, Kanwisher N (1998) A cortical representation of the local visual environment. Nature 392:598-601

Epstein R, Harris A, Stanley D, Kanwisher N (1999) The parahippocampal place area: recognition, navigation, or encoding? Neuron 23:115-125
Epstein R, Graham K, Downing P (2003) Viewpoint-specific scene representations in human parahippocampal cortex. Neuron 37:865-876

Goh JOS, Soon CS, Park D, Gutchess A, Hebrank A, Chee MWL (2004) Cortical areas involved in object, background, and object-background processing revealed with functional magnetic resonance adaptation. J Neurosci 24:10223-10228

Grill-Spector K, Malach R (2001) fMR-adaption: a tool for studying the functional properties of human cortical neurons. Acta Psychol 107:293-321

Grill-Spector K, Kushnir T, Edelman S, Avidan G, Itzchak Y, Malach R (1999) Differential processing of objects under various viewing conditions in the human lateral occipital complex. Neuron 24:187-203

Henderson JM (ed) (2005) Real-world scene perception. Psychology Press, New York

Henderson JM, Ferreira F (2004) Scene perception for psycholinguists. In: Henderson JM, Ferreira F (eds) The interface of language, vision, and action: eye movements and the visual world. Psychology Press, New York, pp 1-58

Henderson JM, Hollingworth A (1999) High-level scene perception. Annu Rev Psychol 50:243-271

Henderson JM, Larson CL, Zhu DC (2006) What makes realworld scenes special? An fMRI study (submitted)

Intraub H (1981) Rapid conceptual identification of sequentially presented pictures. J Exp Psychol Hum Percept Perform 7:604-610

Kaplan S (1992) Environmental preference in a knowledge-seeking, knowledge-using organism. In: Barkow $\mathrm{JH}$, Cosmides $\mathrm{L}$, Tooby $\mathbf{J}$ (eds), The adapted mind: evolutionary psychology and the generation of culture. Oxford University Press, New York, pp 581-598

Kim D, Adalsteinsson E, Glover G, Spielman D (2002) Regularized higher-order in vivo shimming. Magn Reson Med 48:715-722

Kohler S, Crane J, Milner B (2002) Differential contributions of the parahippocampal place area and the anterior hippocampus to human memory for scenes. Hippocampus 12:718-723

Li FF, Van Rullen R, Koch C, Perona P (2002) Rapid natural scene categorization in the near absence of attention. Proc Natl Acad Sci USA 99:9596-9601

Maguire EA (2001) The retrosplenial contribution to human navigation: a review of lesion and neuroimaging findings. Scand J Psychol 42:225-238

Maguire EA, Frith CD, Cipolotti L (2001) Distinct neural systems for the encoding and recognition of topography and faces. Neuroimage 13:743-750

Martinez AM, Benavente R (1998) The AR face database. CVC Tech. Report \#24

Oliva A, Schyns PG (2000) Diagnostic colors mediate scene recognition. Cognit Psychol 41:176-210

Parrish T, Gitelman D, LaBar K, Mesulam M (2000) Impact of signal-to-noise on functional MRI. Magn Reson Med 44:925-932

Potter MC (1976) Short-term conceptual memory for pictures. J Exp Psychol [Hum Learn] 2:509-522

Schyns PG, Oliva A (1994) From blobs to boundary edges: Evidence for time- and spatial-scale-dependent scene recognition. Psychol Sci 5:195-200

Talairach J, Tournoux P (1988) Co-Planar stereotaxic atlas of the human brain: 3D proportional system. Georg Thieme Verlag, New York

Thorpe SJ, Fize D, Marlot C (1996) Speed of processing in the human visual system. Nature 381:520-522

Torralba A, Oliva A (2003) Statistics of natural image categories. Network: Comput Neural Syst 14:391-412 
Vailaya A, Jain A, Jiang Shang H (1998) On image classification: City images vs. landscapes. Pattern Recognit 31:1921-1935

Vessel EA, Biederman I (2002, May) An fMRI investigation of preference habituation. In: Annual meeting of the Vision Science Society, Sarasota

Ward B (2000) Simultaneous inference for fMRI data. Biophysics Research Institute, Medical College of Wisconsin, Milwaukee
Ward B (2002) Deconvolution analysis of fMRI time series data. Biophysics Research Institute, Medical College of Wisconsin, Milwaukee

Wolbers T, Buchel C (2005) Dissociable retrosplenial and hippocampal contributions to successful formation of survey representations. J Neurosci 25:3333-3340 